cited in Chow \& Bukhari Virology 74, $242 ; 1976)$. To apply the model to $\mathrm{Mu}$ it would only be necessary to postulate either that $\mathrm{Mu}$ might be defective in the second set of genes or that $\mathrm{Mu}$ phage with the $G$ segment in the non-viable orientation are able to adsorb to other bacterial strains which have not yet been tested. Further experiments will more completely elucidate the role of $\mathrm{G}$ segment inversion in expression of $\mathrm{Mu}$ genes and will reveal whether inversion of DNA segments comprises a general mechanism of gene control.

\title{
How plants respond to stress
}

\section{from Hamlyn G. Jones}

THE plant hormone abscisic acid (ABA) has been invoked to explain a host of plant processes ranging from dormancy and leaf abscission, through flowering and fruit drop to the response of roots to gravity. However, it is as a stress hormone involved in the droughtinduced closure of stomata, that there is the most complete evidence for a natural role for this hormone. The stomata are the chief control on water loss from plants, so that stomatal closure is an important mechanism enabling plants to survive in dry environments.

There is now evidence that the amount of ABA in tissues of many plant species increases rapidly when the plants are subjected to various stresses, including water deficits. Increased concentrations of ABA can be detected within 7 minutes of the start of a stress period and can increase as much as 40 -fold over 4 hours. The evidence that this stress-induced ABA is involved in at least some of the natural stomatal movements is now overwhelming. Not only is the amount of ABA extractable from leaves generally related to stomatal aperture, but ABA applied to leaves usually results in rapid stomatal closure. Further evidence comes from work by Tal and coworkers at the University of the Negev on a wilty mutant of tomato (flacca) which does not close its stomata. This lesion was found to be due to a deficiency of $\mathrm{ABA}$ which could be overcome by supplying extra hormone.

Two papers in this issue of Nature (Itai et al., page 652 and Itai \& Meidner, page 653 ), provide further evidence of a role for ABA in stomatal physiology. These workers demonstrate, using microautoradiography of leaf or epidermal tissue supplied with ${ }^{14} \mathrm{C}$ labelled ABA, that the hormone can accumulate at the presumed site of action -the stomatal apparatus.

This extends recent work by Loveys (Physiol. Plant. 40, 6; 1977) who showed that although ABA does not appear to be synthesised in the epidermis it does accumulate in the epidermis

Hamlyn $G$. Jones is a Lecturer in the Department of Botany, University of Glasgow. under stress. Nevertheless, there has not yet been an unequivocal demonstration that $\mathrm{ABA}$ accumulates in the stomatal apparatus during natural stress.

If the details of ABA accumulation are not yet proven, the mode of action of the hormone is even less certain. Changes in stomatal aperture are directly caused by changes in the balance between the internal pressure of the guard cells (specialised cells which surround the stomatal pore) and other epidermal cells. The high pressures necessary to open stomata arise from the active accumulation of osmotically active molecules and ions, especially $\mathrm{K}^{+}$. It is likely that ABA acts in some undetermined manner on the accumulation or retention of $\mathrm{K}^{+}$, as discussed by Itai and Meidner.

In spite of these recent successes in explaining stomatal movements in terms of $\mathrm{ABA}$ it is as well to remember that this is not the only mechanism involved in controlling stomatal movements. In the first place, so-called hydroactive closure can begin within a few seconds of changes of leaf water status; a response which is too rapid to involve a hormone such as ABA. Second, the hormone is probably not involved in the normal diurnal opening and closing movements found in most plants. Third, there are several reports of the involvement of other naturally occurring compounds in the regulation of stomatal aperture, particularly under stress. Most evidence has been adduced for an involvement of all-trans-farnesol (a sesquiterpenoid like ABA) which Mansfield's group at Lancaster have reported (Ogunkanmi et al. Planta 117, 293 ; 1974) to accumulate in droughtstressed sorghum and to be effective at closing stomata.

The question still remains as to whether the postulated role for $\mathrm{ABA}$ in promoting stomatal closure is the main role for the apparently excessive quantities of the hormone produced in stress conditions. Many of the known effects of $\mathrm{ABA}$, including reduction in leaf growth or even enhanced leaf senescence (see Milborrow A. Rev. Pl. Physiol. 25, 259; 1974) as well as the reduced proportion of epidermal cells which form stomata and the increased production of epidermal hairs (Quarrie \& Jones J. exp. Bot. 28, 192; 1977), are also well known symptoms of water stress. In the long term, these changes, particularly the reduction in leaf area, may be as important as stomatal closure in reducing water use by plants. Other work (Glinka Pl. Physiol. 51, 217 ; 1973) indicates that ABA can increase the rate of water uptake by plant roots. Taken together, these results suggest that ABA may be involved in a whole syndrome of responses, all of which may contribute to adaptation to stress environments.

Perhaps many of the other reported effects of ABA, such as those on flowering, may also prove to be adaptations to stress environments. Unfortunately much of the evidence on the developmental effects of ABA is contradictory, so there is a clear need for more careful studies of the effects of applied ABA, taking account of the quantities of the hormone getting into the tissue, its distribution and its ultimate fate.

Research on ABA may have important practical consequences. Following the demonstration that some droughttolerant species or varieties produce more ABA than sensitive ones (LarquéSaavedra \& Wain Nature 251, 716; 1974: Ann appl. biol. 83, 291; 1976), several groups around the world are currently screening agricultural crops for ABA production in attempts to breed new varieties capable of high yields in dry regions. Other work concentrates on the use of ABA or its analogues as anti-transpirants to be applied to crops when drought occurs. Much of this work involves the implicit assumption that stomatal closure due to enhanced ABA levels is advantageous in dry situations. Unfortunately the reduction in photosynthesis and harvestable yield which accompanies stomatal closure may outweigh any saving in water, while rapid stomatal closure does not always appear to improve drought tolerance in crops. $\square$

\section{The man-made mouse}

\section{from John K. Heath}

Teratocarcinomas have been the subject of intensive research effort over the past few years. They are malignant tumours composed of 'undifferentiated' embryonal carcinoma stem cells (EC cells) which can differentiate in vivo or in vitro into apparently normal differentiated tissue. Since the cells can grow and

John $K$. Heath is in the Department of Zoology, University of Oxford. 